\title{
The Effect of Patient Acuity Tool on Patient Clinical Practicum Assignments on Nursing Student Satisfaction Levels: A Quasi-Experimental Study
}

\author{
Marionne Leigh V. David ${ }^{1}$, Reeanz Arvin V. Bano ${ }^{1}$, Eric Ferdinand S. \\ Joven ${ }^{1}$, Noel Robertino Manansala ${ }^{1}$, Michaella G. Sta. Ana ${ }^{1}$, Ria Valerie \\ D. Cabanes ${ }^{\star 2} \odot$, Mildred G. Glinoga ${ }^{1}$
}

${ }^{1}$ University of the east ramon magsaysay memorial medical center, Barangay Doña Imelda, Quezon City, Philippines

${ }^{2}$ University of the Philippines Open University, Los Baños, Laguna, Philippines

\section{f OPEN ACCESS \\ Jurnal Keperawatan Padjadjaran (JKP) \\ Volume 9(2), 138-147 (C) The Author(s) 2021 http://dx.doi.org/10.24198/jkp. v9i2.1668 \\ Article Info \\ Received : June 16, 2021 Revised : August 1, 2021 \\ Accepted : August 7, 2021 \\ Published : August 31, 2021 \\ Corresponding author}

Ria Valerie D. Cabanes University of The Philippines Open University, Baños, Philippines, 4031, Phone: 6349 536-6001, E-mail: rdcabanes@up.edu.ph

\section{Citation}

David, M. L. V., Bano, R. A. V., Joven, E. F. S., Manansala, N. R., Ana, M. G. S., Cabanes, R. V. D., \& Glinoga, M. G. (2021). The Effect of Patient Acuity Tool on Patient Clinical Practicum Assignments on Nursing Student Satisfaction Levels: A Quasi-Experimental Study. Jurnal Keperawatan Padjadjaran, 9(2), 138-147. https://doi.org/10.24198/jkp. v9i2.1668

\section{Website}

http://jkp.fkep.unpad.ac.id/index. php/jkp

This is an Open Access article distributed under the terms of the Creative Commons Attribution-NonCommercial 4.0 International License.

\begin{abstract}
Background: Unequal patient assignment is a problem of nursing students during clinical practicum. Registered nurses use patient acuity to ensure balanced work distribution, but its benefits has not been assessed on student nurses to date.

Purpose: The study's objective is to determine the effect of patient acuity tool on student nurses' satisfaction level during practicum.

Methodss: The study utilized quasi-experimental design to level II and III nursing students at a University in Quezon City, Philippines. 6-Module Online course including videos, notes, and quiz using Google Classroom was used; orientation and simulation were conducted through Zoom and Facebook Messenger. The participants were tasked randomly to use Patient Acuity Tool or conventional method during patient distribution. A modified Kuopio University Hospital Job Satisfaction Scale was used to identify and compare satisfaction levels of the two groups. Descriptive and Independent Samples T-test using Statistical Package for the Social Sciences version 26 were used to analyze data.

Results: Requiring factors, motivating factors, and working welfare of students utilizing Patient Acuity Tool are higher in overall mean satisfaction rate $(3.36,3.71,3.89$ vs $3.57,3.81,3.91)$; however, mean satisfaction showed no significant difference based on indicators ( $p$-value $0.372,0.694$, $0.052,0.921$, and 0.469 ) and overall satisfaction ( $p$-value 0.947). The requiring factors of work reflects the lowest mean scores given by those who have used the Conventional Method focusing on number of staff (2.97) and even workload distribution (2.14).

Conclusion: Contrary to the last result, those who have distributed patients using the Patient Acuity Tools gave a mean score of 3.55.
\end{abstract}

Keywords: clinical competency; clinical practicum; nursing students; patient acuity; satisfaction.

\section{Introduction}

Nursing students' clinical experience is considered an essential aspect of nursing education since it molds and prepares their competency in caring for patients (Sharif \& Masoumi, 2005). Although an internship is the best way to learn, some students did not improve their skills despite being in the clinical area (Candelasa, 2016). In upgrading the learning quality, barriers associated with student nurses' knowledge and skillful growth should be assessed; and the quality of care rendered and nurses' productivity are dependent on their job satisfaction (Kousar et al., 2018). A Level II or III student nurse in the Philippines should handle three patients (CHED, 2017). However, some students report lack of direct experience, less opportunity 
to perform practical techniques, not handling complex cases, and the competitive atmosphere and demanding academic requirements make them more unsatisfied (Memarian et al., 2015). Clinical duties are well-rounded as nursing students also spend countless hours studying pathogenesis of a patient's condition, medications, and patient procedures to develop a concrete and holistic care plan (Nurse Journal, 2021).

Student nurses' clinical exposure is significant in enhancing competency. Interweaving theoretical knowledge to these practical settings is one of the challenges during internship. Student nurses need to enhance their reasoning skills as these are salient in the clinical judgment processes (Yauri et al., 2019). According to theory, the combination of practice, good clinical supervision, and equity in workload could enable student nurses to feel competent, useful, and satisfied with their abilities and experiences to take care of the patients (Tiwaken, S., Caranto, L., \& David, 2015). The study was primarily grounded in the Advanced Nurse Practitioner's ComPOSURE Behavior and Patient's Wellness Outcome. The definition of Competence based on the ComPOSURE behavior by (Divinagracia, 2001) can also be applied to nursing students as they are able to gain knowledge and clinical experience followed by expertise in patient care which leads to their satisfaction. In order for an individual to be competent, they must be satisfied with their job. It has been said that job satisfaction affects a person's competency.

Instructors are also key players in maintaining students' satisfaction levels. Since they are at the forefront of clinical training, instructors should guide and actualize students employing both theory and practice through mentoring. Teachers must consider every students' differences - it is not rational to assume that student nurses have similar acquired knowledge and skills because learners have various needs on a given day. A university in Benguet, Philippines, mentioned the importance of reevaluating clinical skills training in the nursing field. Their participants stated some anxiety-provoking problems encountered during practicum, like being handled by a preceptor they are not on good terms with and completing a huge amount of workload in the ward. Each patient's unique requirements either promote or stagnate students' knowledge and skills. Students with fully-loaded procedures can eventually sacrifice theoretical expertise and quality of care. These conditions affect student's satisfaction consequently (Atakro et al., 2019; MacPhee et al., 2017; Tiwaken, S., Caranto, L., \& David, 2015)

Nursing students could distrust their clinical competence due to lack of experience (Hashemiparast et al., 2019). Considering these, it could decrease student nurses' level of satisfaction in the clinical ward and affect their overall performance (Bazrafkan \& Kalyani, 2018; Nurumal, M. S., Diyono, N., \& Che Hasan, 2020). There is a strong correlation between nursing students' satisfaction with their heightened competency (Papastavrou et al., 2016). Results garnered and assessed through the Clinical Learning Environment Supervision and Nurse Teacher $($ CLES $+T)$ show that if students are highly satisfied with the learning environment, most especially its pedagogical facet, burgeoning competencies are established.

The nurses' role is not just limited to providing care in the ward; there are also circumstances when nurses need to transfer patients to different hospital departments which is time-consuming apart from their heavy workload (The Top 3 Benefits of AcuityBased Staffing for Your Organization, n.d., 2014). Deprivation from concentration, an element crucial for a nurse's performance, can happen because of overstretching workload (Safdar et al., 2019). An acuity-based staffing system manages without considering the number of patients. Instead, the system focuses on patient needs. Nurse-patient assignment (allocation of patient care to nurses for a specific shift) is done by charge nurses (Allen, 2015) and faculty members or group leaders on students during clinical learning hours.

By definition, patient acuity is categorizing patients based on the nursing care they require. The Patient Acuity Tool improved nurse's reports on receiving equal assignments with $7 \%$ and $55 \%$ satisfaction before and after patient distribution (Acar \& Butt, 2016). A study of one healthcare corporation claims acuity-based staffing as beneficial in giving fair assignments, matching patient needs and nurses' competence, and increasing nurses' satisfaction and productivity (The Top 3 Benefits of Acuity-Based Staffing for Your Organization, n.d., 2014). However, literature about effectiveness of the Patient Acuity Tool is mostly based on western viewpoint, and its effectiveness to measure level of satisfaction of student nurses concerning patient assignment has not been evaluated to date.

In addition, using the Patient Acuity Tool is linked to increasing nurses' satisfaction (Brennan et al., 2019), better patient outcomes (Nurse Journal, 2021), reduction of stress, decreasing nurse turnover, and highlighting the nurses' exceptional care. The Patient Acuity Tool also benefits the entire healthcare team and the institution (O'Keeffe, 2016).

\section{Methods}

A quasi-experimental design was utilized to determine the effect of using Patient Acuity Tool in clinical practicum patient assignment on the satisfaction level of student nurses. The study was conducted at a University in Quezon City, Philippines. The study aimed to identify and compare the satisfaction levels of nursing students before and after using Patient Acuity Tool and Conventional Methods in assigning patients during clinical practicum. It was hypothesized that the satisfaction levels of those who used the Patient Acuity Tool is significantly higher than those who 
used the Conventional Method.

A modified questionnaire was evaluated by faculty members to assess the appropriateness of the tool. Pilot study was done to regulate the flow of research protocol. The investigators utilized purposive sampling and recruited 60 nursing students from Level II and III using sample size estimation with two means (Sullivan, n.d.). Recruitment was done by posting invitations online and sending invitations through Year Level Group Chats and emails with Class Adviser's approval. Participants were at least 18 years, enrolled in AY 2019-2020, previously assigned in OB-Gyne and Pedia Ward, and with stable internet connection.

The OB-Gyne ward is a mother baby-friendly area with two divisions - the rooming-in unit, which caters to postpartum well mothers and the GYNE for high risk and alterations of the reproductive system. The Pediatric ward consists of a clean isolation room, which caters to cancer patients undergoing chemotherapy; a dirty isolation room where patients with communicable diseases are being accommodated; and the general ward for other pediatric cases. The OB-gyne Ward and Pediatrics Ward cater to 20 and 18 patients, respectively. During the clinical practicum, 10-12 students are assigned in these wards, handling 1-2 patients on an 8-hour shift. In addition, Department rotation happens every two weeks for students taking Care of Mother, Child and Family (Well-clinic) and every four weeks for students taking Care of Mother and Child at Risk or with acute and chronic problems.

Participants were excluded if they could not attend the orientation and were absent for more than two days during clinical practicum, either excused or not. The recruitment happened for a week. An orientation was given to the participants and they were asked to sign an informed consent and upload them to the Google Classroom before the online course was conducted.

In line with the recent announcement of extended Enhanced Community Quarantine in Luzon (Memorandum from the Executive Secretary On Community Quarantine Over the Entire Luzon and Further Guidelines for the Management of the Coronavirus Disease 2019 (COVID-19) Situation, 2020), the investigators conducted all data collection process online.

\section{Intervention protocol}

The investigators developed a 6-Module Online Series about Patient Acuity Tool orientation. Each module includes video, lecture note, and quiz. Participants can only proceed to the next module if they attained a passing score of $70 \%$ in modular quizzes. Google Classroom, Office 365, and official university email accounts were utilized to recruit, communicate with the participants, provide lecture series, collect answered research instruments, and disseminate results. The investigators facilitated all data collection procedures as supervised by Faculty members.
The entire learnings of the student nurses can be applied in simulated online clinical practice scenarios to enhance their self-confidence and clinical judgment skills because they can do procedures repetitively, which allows thorough practice that provides no risk to patients since training is webbased simulated (Hustad et al., 2019).

The participants were divided into control and experimental groups using SPSS randomizer, the investigators who conducted the simulation were also randomized. Furthermore, the two groups were divided into A and B for OB-Gyne and Pediatric wards. The intervention consisted of utilizing the Patient Acuity Tool on patient assignment on their simulated OB-Gyne and Pediatrics ward rotation. The experimental group received the Patient Acuity Tool intervention, and the control group used a conventional method (randomized) of patient assignment. A student leader was randomly appointed per group and was tasked to assign patients to the members. After receiving their patient assignments, the group members individually computed patient acuity scores of the given case scenarios to evaluate if there was a fair distribution of patients and tasks to everyone. The online simulation was done through Zoom Conference and Facebook Messenger Chat. The lecture part can be accomplished for 4-6 hours, quizzes for 1-3 hours. This was done during the first day. The simulation and post-test using the Kuopio University Hospital Job Satisfaction Scale (KUHJSS) took 3 hours, conducted the following day. Google Forms were utilized and names were not required in filling up the questionnaire. Collection of data was automatically done thru Excel as a feature of Google Forms. Students who completed the study received a certificate of participation.

Figure 1 shows that a total of 63 participants enrolled in the Google Classroom. However, there were two participants who did not finish the simulation and submit the post-test questionnaire (due to unexpected internet connectivity problem).

\section{Research Tools}

There are five Patient Acuity Tool acuity categories namely procedures that are complicated, education, interventions given (psychosocial or therapeutic), drugs administered, and complex IV drugs and measurements. Rating options ranged from 1 (lowest) to 4 (highest). These ratings are then added to get the sum of the acuity score of an individual patient. Total score ranges from one (1) to sixty (60). Grouping of patients follows as all acuity scores are categorized from 1 to 4 (The Top 3 Benefits of AcuityBased Staffing for Your Organization, n.d.). Patients tagged within categories are carefully assigned to students to distribute the workload equally (e.g., a student will handle 2 patients under category 1 and 3,2 patients under category 2, or just 1 category 4 patient). With Cronbach's alpha coefficient of 0.87 , Patient Acuity Tool has been identified to be reliable and valid over the years (Perroca, 2011). Validity of 

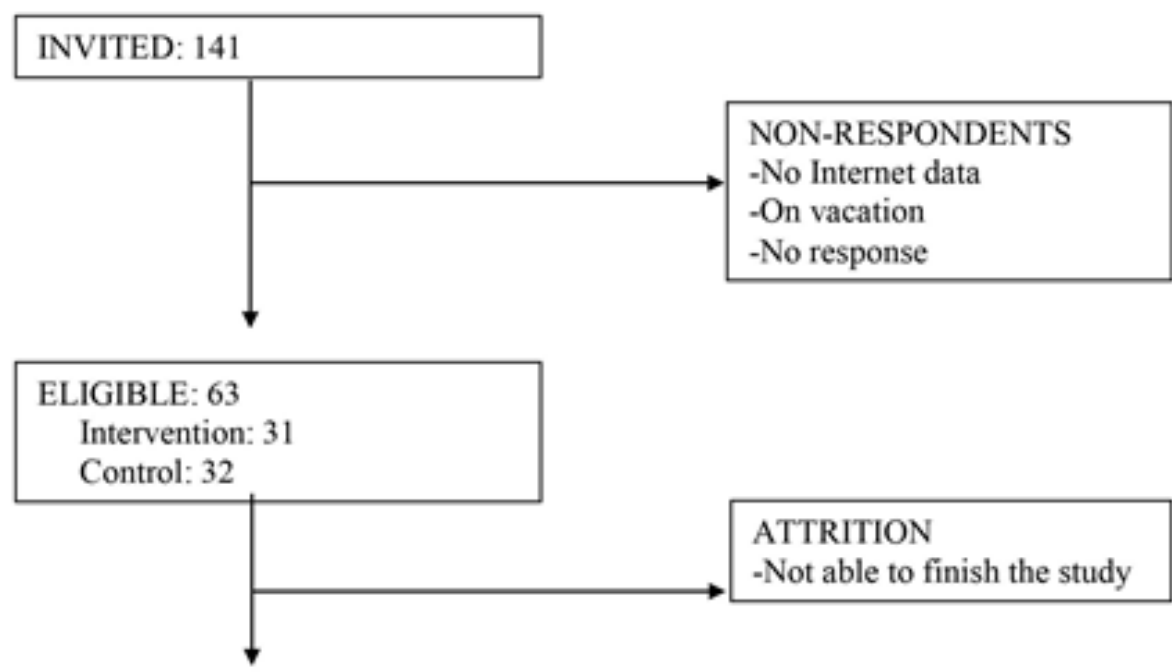

COMPLETED : 61

Figure 1. Participant Flow

the constructs was also examined. The variables used to formulate the instrument were essential and included on actual discrimination of patients' care needs and categories. The KUHJSS assessment was invented at the Department of Nursing Science of the University of Eastern Finland (Kvist et al., 2012). Ten items identify the demographic profile and professional traits; and thirty-seven (37) 5-point Likert scale to determine job satisfaction levels.

The data analysis used Statistical Package for the Social Sciences (SPSS) version 26. It used descriptive statistics (percentage distribution and frequency of demographic profile and inferential statistics) and Independent Samples T-test to identify mean differences between groups.

The study received approval from the Institutional Ethics Review Committee. Strict compliance with ethical protocols was done. Informed consents were explained, signed, and submitted through Google Classroom. Three Faculty members supervised the entire process. Authorization to use the tools in this study was granted by the authors.

UERMMMCI Ethics Review Committee has approved this study (0781/C/2020/009). Informed consents were signed and submitted to investigators upon enrolling in the Google Classroom.

\section{Results}

Table 1 shows a total of 61 students participated in this study; 31 participants were assigned to use the Patient Acuity Tool while 30 were assigned to use the conventional method. Participants are mostly females (93\% and $80.64 \%)$ and there were more Level II students in both groups $(83.30 \%$ and $67.74 \%$ respectively).

All the answers of the participants who have utilized the Patient Acuity Tool, on the other hand, are all under 'partly agree'. This is similar to the answer of those who used the Conventional Method except for Requiring Factors of Work which is interpreted as 'neither agree nor disagree' as seen in Table 2. Requiring factors of work, motivating factors of work, and working welfare of those who used the Patient Acuity Tool are higher in overall mean satisfaction rate in terms of individual indicators.

Independent samples t-test was calculated to compare these five indicators between control and experimental groups. The mean of the first, second, and fourth indicators (Requiring Factors of work, motivating factors of work, and working welfare) is higher on the experimental group than the control group with absolute values of $t$-scores reveal that t-obs $(-0.899,-0.276$, and -0.100$)$ which leads to failure of rejecting null. Although there was an increase in the level of satisfaction of these three factors, no significant differences were noted on all indicators.

Table 4 shows $t$ observed of 0.067 . With a degree of freedom of 46 and a level of significance of 0.05 on a two-tailed test, it shows that our critical $T$ value is $|1.671|$. T observed is less than t critical; no significant difference between participants' satisfaction level using Conventional Method and Patient Acuity Tool was noted.

\section{Discussion}

This study noted that there is no significant difference between overall satisfaction levels of students who used the Patient Acuity Tool and Conventional Method ( $p$-value 0.947). Three indicators, namely requiring factors of work; motivating factors of work; and working welfare; showed an increase in the mean satisfaction level. However, p-values show that there are no significant value between the 
Table 1. Summary of Demographic Profile of Respondents

\begin{tabular}{lcccc}
\hline \multirow{2}{*}{ Variable } & \multicolumn{2}{c}{ Conventional } & $\mathbf{n}$ & PAT \\
\cline { 2 - 5 } & $\mathbf{n}$ & $\%$ & & $\%$ \\
\hline Gender & 2 & 6.66 & 6 & 19.35 \\
Male & 28 & 93 & 25 & 80.64 \\
Female & & & 21 & 67.74 \\
Year Level & 25 & 83.3 & 10 & 32.25 \\
2 & 5 & 16.66 & & \\
3 & & & 15 & 48.38 \\
Ward & 15 & 50 & 16 & 51.61 \\
OB-Gyne & 15 & 50 & & \\
Pedia & & 50 & \\
\hline
\end{tabular}

Patient Acuity Tool (PAT); Obstetrics-Gynegology (OB-Gyne)

different indicators of student satisfaction ( 0.372 , $0.694,0.052,0.921$, and 0.469 ). The analysis of these findings are highlighted in the succeeding individual discussions of the different indicators.

Satisfaction of students in terms of Requiring Factors of Work using Conventional Method has an overall weighted mean of 3.36 , interpreted as "Neither agree nor disagree." Student nurses' satisfaction in requiring factors of work which received patients using Patient Acuity Tool has an overall weighted mean of 3.57 translated to "Partly agree" on this certain domain. Nurses evaluated the practice environment to determine positive and stimulating aspects of their day-to-day practice; this can vary depending on the nurse's country (Kvist et al., 2012). The challenges that nurses' face every day can be overcome by appropriateness of resources for nurses.

The importance of measuring appropriateness of workload of nurses is not only exclusive in the Philippines. The differences based on culture, workforce, and resources available in the work environment should be considered. Since student nurses are not yet receiving salaries or benefits, national policies on nurses do not influence the answers yet. Level II and III students are still very excited to experience their clinical exposure, which is a new experience for them. The orientation and basic tasks that they need to accomplish are not too stressful for them as the excitement and eagerness to learn overpower the demands of their tasks. This has also been balanced because compared to Level III students, the Level II students do not have too much paperwork to accomplish, and their subjects are considered lighter. Hence, there is still a good balance between work and personal life for the younger participants. Another balancing factor is that Level II students are also allowed to work in teams and pairs, giving them higher sense of satisfaction during clinical duty even if they have more patients than the others; this is in comparison to Level III students who are transitioning towards concepts needing higher skills as well as leadership and management.

Factors associated with work satisfaction for registered nurses. Nurses' quantitative workload was not related to job satisfaction. Nurses who think they have a high or low workload have no difference in level of satisfaction because of perceived fairness or "distributive justice" of workload (Ruggiero, 2005). Furthermore, satisfaction is found not to be affected if everyone is working hard. This is what Patient Acuity Tool tries to address, distributing patients based on tasks and not quantity.

Decreased concentration due to overloading of work creates dissatisfaction (Safdar et al., 2019). Dissatisfaction comes from lack of justice in terms of workloads, few days off, and influences on recruitment and retention of nurses in ward; meanwhile, newly graduates get dissatisfied with the salary (Cowin, 2002). Other factors that influence the satisfaction of newly grads include professional status and autonomy. Considering that most of the nurses in hospital nowadays are new graduates, and the study participants are incoming level III and graduating students; understanding these factors can help educators identify issues regarding their workplace satisfaction in the future. These identified concerns can be used to orient students and help them identify solutions in advance.

Satisfaction of students who used the Conventional Method of patient assignment in terms of Motivating Factors of Work has an overall weighted mean of 3.71 , interpreted as a "Partly agree" statement. Students who used the Patient Acuity Tool on patient assignment had a partial difference in overall mean scores with 3.81. Students could appreciate their tasks because of the fulfilling nursing profession has to offer in their job experience (Obrist, R., Berger, D. P., \& Obrecht, 1990). Nurses working in a hospital for longer than ten years appreciate their work more than new graduates (Cowin, 2002; Rambur et al., 2005). Nurses who can apply their skills and expertise are more satisfied. The student nurses only have 3 years of clinical experience, half of which is intensive practice; this is not enough to fully recognize their tasks yet as established in the previous study. They still see their assignments and clinical duties as interesting and suitably challenging. Fresh graduates have a very 
Table 2. Mean Scores per Survey Question from the Modified KUHJSS of students using Conventional Method and PAT

\begin{tabular}{lcc}
\hline & $\begin{array}{c}\text { CONVENTIONAL } \\
\text { Weighted Mean }\end{array}$ & $\begin{array}{c}\text { PAT } \\
\text { Weighted Mean }\end{array}$ \\
\hline & Requiring Factors & of Work \\
My workload is appropriate & 3.87 & 3.97 \\
There are usually enough staff in my unit & 2.97 & 3.29 \\
I do not find my work too stressful & 3.74 & 3.42 \\
I am satisfied with my working hours & 3.84 & 3.68 \\
Combining work and personal life is successful & 3.64 & 3.52 \\
The workload is distributed evenly in my unit & 2.14 & 3.55 \\
Overall & 3.36 & 3.57
\end{tabular}

Motivating Factors of Work

My work is interesting

I appreciate my own work

I can apply a wide range of my skills and expertise in my work

My work tasks are suitably challenging

Overall

\begin{tabular}{|c|c|c|}
\hline \multicolumn{3}{|c|}{ Working Environment } \\
\hline My unit has appropriate equipment to ensure quality of care & 4.17 & 3.68 \\
\hline My work unit is comfortable & 3.94 & 3.58 \\
\hline My work unit is safe and secure & 4.36 & 3.77 \\
\hline Overall & 4.17 & 3.68 \\
\hline
\end{tabular}

I look after my personal well being

\section{Working Welfare}

I am happy with my current health

$3.9 \quad 4.10$

I am active in developing myself professionally

I feel I am a competent student nurse

idealistic mind that tends to get culture-shocked when they start working. New graduates are constantly looking for career advancement; most become impatient and transfer from one hospital to another. Hospital administrators are aware of the new challenges, the incoming generation of hospital workforce face gives them ample time to prepare solutions and programs to increase retention.

Satisfaction of students in terms of Working Environment has an overall weighted mean of 4.17 for students who didn't receive intervention interpreted as a "Partly agree" statement compared to the overall means of 3.68 of the group who used an acuity tool. Student nurses do not only focus on the tasks they have to do during their clinical exposure. The working environment including the availability of needed medical equipment, having ample space to work on their nursing responsibilities and paperworks, good ventilation, comfort, and safe surroundings matter to these young minds as well. Technology that can promote healthcare efficiency, safety, quality, and cost (Rajeswaran, 2016) greatly influences workplace satisfaction as part of the care the students can provide for their patients. This aspect is also challenging for hospitals in the developing world. A study aligned staff satisfaction directly with organizational support provided by hospitals on factors like identifying and implementing proactive strategy to reduce adverse events, nurses' decreased job satisfaction and emotional exhaustion. This then necessitates 
Table 3. Significant Difference between Level of Satisfaction of Students using Conventional Method and PAT based on indicators

\begin{tabular}{lllcc}
\hline \multicolumn{1}{c}{ Indicators } & \multicolumn{1}{c}{ Tool } & Mean & t-value & p-value \\
\hline Requiring Factors of Work & Conventional & 3.36 & -0.899 & 0.372 \\
& Acuity & 3.57 & & 0.694 \\
\hline Motivating Factors of Work & Conventional & 3.71 & -0.376 & \\
& Acuity & 3.81 & & 0.052 \\
Working Environment & Conventional & 4.17 & & 0.921 \\
& Acuity & 3.68 & -0.100 & \\
Working Welfare & Conventional & 3.89 & & 0.469 \\
& Acuity & 3.91 & 0.728 & \\
Sense of Community & Conventional & 4.02 & 3.85 & \\
& Acuity & &
\end{tabular}

Table 4. Difference of Total Satisfaction Scores of Student Nurses after using PAT and Conventional Method of Patient Assignment

\begin{tabular}{cccccccc}
\hline Test & \multicolumn{3}{c}{ Control } & \multicolumn{2}{c}{ Experimenal } & \multicolumn{3}{c}{ t-test } \\
\cline { 2 - 8 } & Mean & SD & Mean & SD & $\mathbf{t}$ & dF & $\mathbf{p}$ \\
\hline $\begin{array}{l}\text { Modified } \\
\text { KUHJSS }\end{array}$ & 3.75 & 0.51 & 3.74 & 0.96 & -0.067 & 46 & 0.947 \\
\hline
\end{tabular}

leaders to focus on improving the work environment, which significantly affects nurse workloads and satisfaction. These factors may not be touched since the investigators have used digital simulation of their clinical rotation.

Satisfaction of students in terms of Working Welfare has an overall weighted mean of 3.89 interpreted as a "Partly agree" statement, in comparison with students who underwent intervention, overall mean showed a slight difference, 3.91. There is a vast amount of literature on the causes of anxiety in nurse education (PowellCope et al., 2008). It was recorded that compared to other health fields and the general population; student nurses have a higher level of stress (Pryjmachuk \& Richards, 2007). Competence can also influence student's working welfare as they see themselves developing professionally. When nurses can apply their skills and expertise in care for the patients, they are more satisfied. This is congruent to another study where participants improved safety and effectiveness of the work they perform because of competency-based management wherein talents and skills can be applied to wider and broader range in clinical areas (Nurumal et al., 2020b; Por et al., 2011). A study showing $50 \%$ of participants recognizing themselves as competent and $47.8 \%$ of student nurses particularly competent in the clinical setting noted that fewer students in the clinical area could lead to higher competence (Sherman \& Eggenberger, 2009). Proper assignment of clients per student can greatly influence their competence and eventually their satisfaction. Level II students are still in their phase of adjustment, and if the students feel that they are given the right number of tasks and patients that can improve their skills significantly, they can appreciate the clinical experience more. This can make them look forward to the next rotation as they perceive it as an excellent opportunity to gain more knowledge. There are more responsibilities and skills needed from Level III. However, this also gives them a greater sense of competence and prepares them for a higher learning degree.

Satisfaction of students in terms of Sense of Community has an overall weighted mean of 4.02 , interpreted as a "Partly agree" statement; students who used the Patient Acuity Tool showed a slight decrease in mean scores with 3.85. Participants were able to apply various skills in the ward if there is a balanced workload rather than giving all workload to the one with the most experiences (PowellCope et al., 2008). It was noted that collaborative construction of knowledge and verbalizing thought processes which can be gained during a contextualized learning experience can benefit a student's clinical reasoning skills (Yauri et al., 2019), granting students the ability to learn by watching their classmates perform procedures and trusting their classmates' expertise, it does not give the same satisfaction if they are doing the procedures themselves. The satisfaction can also be influenced by the strong teamwork of the level II and III batch, as seen in their curricular and extracurricular activities. Helping one another academically is also a common observation with the participants. These students have community spirit, and they also have a constant venue for keeping each other updated. Regardless, nurses demonstrated high satisfaction 
in mentoring students but confessed to viewing this as extra work which can lower satisfaction.

The difference between total satisfaction scores of student nurses who used Patient Acuity Tool and Conventional Method is not significant. Similarly, workload and management tools like acuity-based staffing are not directly associated with staff satisfaction (Oermann, 1999). Massive workload, shortage in hospital staffing, busy wards, and excessive students' requirements are considered hindrances in students' training, affecting satisfaction.

\section{Conclusion}

Contrary to a number of studies showing significant effects on nurses' overall satisfaction levels, the use of Patient Acuity Test is noted to have no effects on satisfaction. The results of the patient satisfaction yielded higher in terms of requiring factors of work, motivating factors of work, and working welfare to the participants who used the Patient Acuity Tool during the simulation of patient distribution.

While the results showed an insignificant overall difference, these results could improve specific aspects of patient distribution, eventually enhancing students' satisfaction and learning methods. The common method of getting equal number of patients without considering the number of procedures done should be re-strategized and using the Patient Acuity Tool can address this issue. This shall give a new light on patient distribution highlighting that equity should be best considered over equality. The lowest score of 2.14 in the statement 'The workload is distributed evenly in my unit' given by those who have used the Conventional Method, compared to the 3.55 means score of those who utilized the Patient Acuity Tool, reflects an improvement in the main concern of this study.

The higher satisfaction levels in 3 individual indicators provide a promising foundation of the Patient Acuity Tool in promoting requiring factors, motivating factors, and most especially working welfare of nursing students, can be take into account to improve clinical rotation experience and satisfaction level.

The complex use of Patient Acuity Tool and the minimal time to learn and adapt to its use probably impacted the satisfaction levels. Nonetheless, the investigators recommend nursing educators to give an opportunity the use of Patient Acuity tool during patient distribution when students are conducting their clinical practicum.

The disruption of academic curriculum, which led the investigators to conduct web-based simulation might have also significantly eliminated important factors which only an actual clinical experience can provide. The faculty and administrators are encouraged to conduct further studies to assess Patient Acuity Tool's effects in implementing guidelines for assigning patients to students in an actual clinical setting.
Future researchers should consider Patient Acuity Tool's use across different year levels and in assigning patients in other clinical areas. A pretestposttest design may be used for broader perspective or measurement of participants' level of satisfaction.

\section{References}

Acar, I., \& Butt, S. E. (2016). Modeling nursepatient assignments considering patient acuity and travel distance metrics. Journal of Biomedical Informatics, 64, 192-206. https:// doi.org/10.1016/j.jbi.2016.10.006

Allen, S. B. (2015). The nurse-patient assignment: Purposes and decision factors. Journal of Nursing Administration, 45(12), 628-635. https://doi.org/10.1097/ NNA.0000000000000276f

Atakro, C. A., Armah, E., Menlah, A., Garti, I., Addo, S. B., Adatara, P., \& Boni, G. S. (2019). Clinical placement experiences by undergraduate nursing students in selected teaching hospitals in Ghana. BMC Nursing, 18(1), 1-10. https:// doi.org/10.1186/s12912-018-0325-8

Bazrafkan, L., \& Kalyani, M. N. (2018). Nursing students' experiences of clinical education: A qualitative study. Investigación y Educación En Enfermería, 36(3), e04-e04. https://doi. org/10.17533/udea.iee.v36n3a04

Brennan, C. W., Krumlauf, M., Feigenbaum, K., Gartrell, K., \& Cusack, G. (2019). Patient acuity related to clinical research: Concept clarification and literature review. Western Journal of Nursing Research, 41(9), 1306-1331. https:// doi.org/10.1177/0193945918804545

Candelasa, L. (2016). Related learning experience performance of nursing students and practices of clinical instructors on three-day and five-day schemes.

CHED. (2017). CHED Memorandum Order No. 15. Policies, Standards, and Guidelines for Bachelor of Science in Nursing (BSN) Program. Retrieved December 21, 2020, from https:// ched.gov.ph/wp-content/uploads/2017/10/ CMO-15-s-2017.pdf

Cowin, L. (2002). The effects of nurses'job satisfaction on retention: An Australian perspective. Journal of Nursing Administration, 32(5), 283-291. https://doi.org/10.1097/00005110-20020500000008

Divinagracia, C. (2001). Advanced nurse practitioners' COMPOSURE behaviors and patients' wellness outcome. UERMMMC (University of the East Ramon Magsaysay Memorial Medical Center) Journal of Health Sciences, 4(2), 50-53.

Hashemiparast, M., Negarandeh, R., \& Theofanidis, D. (2019). Exploring the barriers of utilizing theoretical knowledge in clinical settings: A qualitative study. International Journal of Nursing Sciences, 6(4), 399-405. https://doi. org/10.1016/j.ijnss.2019.09.008. 
Hustad, J., Johannesen, B., Fossum, M., \& Hovland, O. J. (2019). Nursing students' transfer of learning outcomes from simulation-based training to clinical practice: $A$ focus-group study. BMC Nursing, 18(1), 1-8. https://doi. org/10.1186/s12912-019-0376-5

Kousar, S., Hussain, M., Afzal, M., Gilani, P. A., \& Azhar, M. (2018). Impact of job satisfaction on nurses' performance. Saudi Journal of Nursing and Health Care, 1(1), 49-55. https://doi.org/ 10.21276/sjnhc

Kvist, T., Mäntynen, R., Partanen, P., Turunen, H., Miettinen, M., \& Vehviläinen-Julkunen, K. (2012). The job satisfaction of Finnish nursing staff: The development of a job satisfaction scale and survey results. Nursing Research and Practice, 2012, 1-11. https://doi. org/10.1155/2012/210509

MacPhee, M., Dahinten, V., \& Havaei, F. (2017). The impact of heavy perceived nurse workloads on patient and nurse outcomes. Administrative Sciences, 7(1), 7. https://doi.org/10.3390/ admsci7010007

Memarian, R., Vanaki, Z., \& Baraz, S. (2015). Learning challenges of nursing students in clinical environments: A qualitative study in Iran. Journal of Education and Health Promotion, $4(1), \quad 52 . \quad$ https://doi.org/10.4103/22779531.162345

Memorandum from the Executive Secretary On Community Quarantine Over the Entire Luzon and Further Guidelines for the Management of the Coronavirus Disease 2019 (COVID-19) Situation. (2020). Official Gazette of the Republic of the Philippines. Retrieved December 10, 2020, from https:// www.officialgazette.gov.ph/2020/03/16/ memorandum-from-the-executive-secretaryon-community-quarantine-over-the-entireluzon-and-further-guidelines-for-themanagement-of-the-coronavirus-disease2019-covid-19-situation/.

Nurse Journal. (2021). Common Courses You Will Take For Your RN Degree. Retrieved January 6, 2021 https://nursejournal.org/articles/ common-courses-you-will-take-for-your-rndegree/

Nurumal, M. S., Diyono, N., \& Che Hasan, M. K. (2020a). Self-efficacy levels regarding interprofessional learning skills among undergraduate healthcare students in Malaysia. sultan Qaboos University Medical Journal, 20(4), e374-e379. https://doi. org/10.18295/squmj.2020.20.04.015

Nurumal M. S., Sabran, N. M., Hamid, S. H. A., \& Hasan, M. K. C. (2020b). Nurses' awareness on patient safety culture in a newly established university hospital. Jurnal Keperawatan Indonesia. https://doi.org/10.7454/jki. v0i0.1088

O'Keeffe, M. (2016, September). Practical steps for applying acuity-based staffing. American
Nurse Today. Retrieved December 18, 2020 from https://www.myamericannurse.com/wpcontent/uploads/2016/09/ant9-ACUITY-817. pdf

Obrist, R., Berger, D. P., \& Obrecht, J. P. (1990). Attitude of ambulatory oncological patients to academic medicine, their physician and nurse. Results of an anonymous survey. Schweiz Rundsch Med Prax, 79(14), 416-419. https:// pubmed.ncbi.nlm.nih.gov/2343227/

Oermann, R. (1999). Behavioral Objectives-Evaluation in Nursing. Springer.

Papastavrou, E., Dimitriadou, M., Tsangari, H., \& Andreou, C. (2016). Nursing students' satisfaction of the clinical learning environment: a research study. BMC Nursing, 15(44). https:// doi.org/10.1186/s12912-016-0164-4

Perroca, M. G. (2011). Development and content validity of the new version of a patient classification instrument. Rev. Latino-Am. Enfermagem, 19(1). https://doi.org/10.1590/ S0104-11692011000100009

Por, J., Barriball, L., Fitzpatrick, J., \& Roberts, J. (2011). Emotional intelligence: Its relationship to stress, coping, well-being and professional performance in nursing students. Nurse Education Today, 31(8), 855-860. https://doi. org/10.1016/j.nedt.2010.12.023

Powell-Cope, G., Nelson, A. L., \& Patterson, E. S. (2008). Patient care technology and safety. In R. G. Hughes (Ed.), Patient safety and quality: An evidence-based handbook for nurses. Agency for Healthcare Research and Quality.

Pryjmachuk, S., \& Richards, D. A. (2007). Predicting stress in pre-registration nursing students. British Journal of Health Psychology, 12(1), 125-144. https://doi. org/10.1348/135910706X98524

Rajeswaran, L. (2016). Clinical experiences of nursing students at a selected institute of health sciences in Botswana | Insight Medical Publishing. Health Science Journal. https://doi. org/10.21767/1791-809x.1000471

Rambur, B., Mclntosh, B., Palumbo, M. V., \& Reinier, K. (2005). Education as a determinant of career retention and job satisfaction among registered nurses. Journal of Nursing Scholarship, 37(2), 185-192. https://doi.org/10.1111/j.15475069.2005.00031.x

Ruggiero, J. S. (2005). Satisfaction Among Nurses. Work, 35(5), 254-263.

Safdar, A., Susilaningsih, F. S., \& Kurniawan, T. (2019). Relationship between workload performance and job satisfaction. Jurnal Keperawatan Padjadjaran, 7(3). https://doi. org/10.24198/jkp.v7i3.1178

Sharif, F., \& Masoumi, S. (2005). A qualitative study of nursing student experiences of clinical practice. BMC Nursing, 4(1), 1-7. https://doi. org/10.1186/1472-6955-4-6

Sherman, R., \& Eggenberger, T. (2009). Taking charge: What every charge nurse needs to 
know. Nurses First. The Official Journal of the CenterforAmerican Nurses, 2(4), 6-10. https:// www.emergingrnleader.com/wp-content/ uploads/2012/06/NursesFirst09August.pdf

Sullivan, L. (2021). Power and Sample Size Determination. Retrieved June 12, 2021, from https://sphweb.bumc.bu.edu/otlt/mphmodules/bs/bs704_power/bs704_power_print. html

The Top 3 Benefits of Acuity-Based Staffing for Your Organization. (2014). API Healthcare. Retrieved June 12, 2021, from https://www. slideshare.net/APIHealthcare/benefits-acuity- basedstaffingapproved-jb20422usi

Tiwaken, S., Caranto, L., \& David, J. (2015). The Real World: Lived Experiences of Student Nurses during Clinical Practice. International Journal of Nursing Science, 5(2), 66-75. https://doi.org/ 10.5923/j.nursing.20150502.05

Yauri, I., Nash, R., \& Ramsbotham, J. (2019). Improving Student Nurses' Clinical-Reasoning Skills: Implementation of a Contextualised, Guided Learning Experience. Jurnal Keperawatan Padjadjaran, 7(2). https://doi. org/10.24198/jkp.v7i2.1166 